\title{
Phase II Clinical Trial to Establish Efficacy of a Locally Appropriate Bivalent Anti Snake Venom in Pakistan
}

\author{
Naeem Quraishi ${ }^{1, *}$, Tasneem Ahmad ${ }^{1}$, Allah-Bux Ghanghro ${ }^{2}$, Abdullah Arejo $^{3}$, \\ Sharib S. Muhammad ${ }^{1}$ and Atta Chandio ${ }^{4}$ \\ ${ }^{1}$ ASVIARV Serology Laboratory, Peoples Medical University Nawabshah, Pakistan \\ ${ }^{2}$ Institute of Biochemistry, Sindh University Jamshoro, Pakistan \\ ${ }^{3}$ Parasitology Department, Sindh Agriculture University, Tando Jam, Pakistan \\ ${ }^{4}$ Faculty of Community Medicine \& Public Health Peoples University of Medical \& Health Sciences for Women \\ Nawabshah, Pakistan
}

\begin{abstract}
Objective: This study was conducted to determine the efficacy of Snake anti-venom Immunoglobulin [IgG] manufactured by Anti-Snake Venom [ASV]/Anti-Rabies [ARV] Serology Laboratory, Health Department, Government of Sindh.

Methods: The prospective, observational single arm study was conducted after the approval of IRB. Study included six patients with viper [Echis carinatus sochureki] snakebites referred to the emergency ward of Peoples University of Medical \& Health Sciences Hospital, Nawabshah and District Headquarter Hospital Mithi, Sindh, Pakistan with consultation of Clinical and Principal investigator. The study was conducted over a period of three months [August 2015 to November 2015]. All patients were given IV infusion of $10 \mathrm{~mL}$ [1 vial] investigational ASV diluted in $100 \mathrm{~mL}$ normal saline except one patient who received $5 \mathrm{~mL}$ management dose and $5 \mathrm{~mL}$ subsequent dose for the recovery of coagulopathy. The efficacy was assessed by Primary and secondary efficacy endpoints, i.e. the dose at which maximum no of patients were treated [permanent restoration of normal blood coagulation tested by 20-minute whole blood clotting test [20-minute WBCT] with minimum toxicity.
\end{abstract}

Results: All patients recovered from coagulopathy after receiving IV infusion of $10 \mathrm{~mL}$ investigational ASV diluted in 100 $\mathrm{mL}$ normal saline tested by 20-minute WBCT. Mean Recovery time was 9:15 $\pm 3: 25$ hours.

Conclusion: Safety and efficacy was assessed for the Bivalent Anti venom Immunoglobulin-NQ1 [IgG] manufactured by ASVIARV Serology Laboratory, Health Department, Government of Sindh.

Keywords: Immunoglobulin [lgG], anti-snake venom [ASV], 20-minute WBCT, Echis carinatus sochureki [Lundi], Coagulopathy.

\section{INTRODUCTION}

Snakes have a worldwide distribution excluding Arctic, New Zealand and Ireland with a denser inhabitancy in tropical and temperate countries [1,2]. Although of the 3,000 snake species found worldwide, only $25 \%$ are considered dangerous and, most of the bites even those delivered by venomous species are innocuous. Envenoming by snake bite is a common life threatening medical emergency that severely affects the locally bitten area as well as multiple organ system $[3,4]$. Despite the fact that a large number of these victims survive, permanent physical sequel arising from local tissue necrosis and psychological sequel put considerable impact on the economy of the families and states as most snakebite victims are young [5].

*Address correspondence to this author at the ASVIARV Serology Laboratory, Peoples University of Medical \& Health Sciences, B-23 LMC Colony, Jamshoro, Sindh, Pakistan; Tel: +92 3003241478 ;

E-mail: naimquraishi@yahoo.com
As Snakebite is largely a problem of the rural area and reliable data for incidence, morbidity and mortality are scarce [6]. There are approximately 100,000 deaths per year globally caused by snakebites which mostly involve the inhabitants of resource -poor rural areas [7]. The highest number of annual cases of envenoming occur in South Asia (estimated to be $1,210,000)$ followed by South East Asia $(1,11,000)$ and East Sub-Saharan Africa $(43,000)$ [2].

There are some 57 land snake species in Pakistan in which thirteen 13 are venomous. Commonest are cobra, krait, Saw scaled and Russell viper [8]. It is estimated that here the annual death rate because of snake envenoming is around 1.9 per 100,000 populations, regarding Sindh province, it is among the five commonest causes of admission to the hospitals as depicted by the records of secondary health care centres [9] with regional immunoglobulins in the types of snakebite.

(c) 2017 Lifescience Global 
In Sindh, seven venomous species are present in desert/ arid zone, out of which six are of medical importance. The saw scaled viper Echis carinatus sochureki (Lundi) is diffusely spread over the entire province with highest concentration in desert area [9]. Snake venom consists of a complex mixture of toxins and enzymes, each of which has a distinct toxicological action. South Asian viperidae snakes envenoming results in localized pain and tissue damage at bitten area manifesting as swelling, blistering, bleeding, and necrosis of the area sometimes extending to the whole limb [10]. It can also induce systemic coagulopathy and platelet dysfunction, leading to spontaneous haemorrhages and continual bleeding from fang marks, wounds, or gums. Visceral bleeding leads to the complication, intracranial anterior pituitary haemorrhage and multi-organ failure that are common causes of death [11].

The victims of snakebites mainly belong to rural population, who are bitten during fieldwork and while sleeping outdoors [12]. Coagulopathy is a common manifestation of echis carinatus envenoming [13]. Coagulopathy after echis carinatus snakebite is confirmed by 20-minute whole blood clotting test (20minute WBCT), usually applied in the local setting as per hospital protocol. In a study of snakebite cases conducted in Liaquat University Hospital Hyderabad/Jamshoro, Sindh, $95 \%$ of cases were caused by viper's bite (hemotoxic) having haemostatic abnormalities [14].

The only definite treatment for snakebite envenoming is immunotherapy by anti-snake venom (ASV), also known as anti-venin and anti-snake venom serum [15]. These Anti-venoms are produced by the plasma fractionation of immunized animals, usually horses [16].
Depending on the number of snake species (single or multiple, respectively) whose venoms are used for animal immunization, anti-venin can be either monovalent or polyvalent. while monovalent antivenom is often more efficacious, the preference is given to the production of polyvalent anti-venom in many countries as in most cases biting snake species identification is generally unattainable for the attending physician.

The treatment success of anti-venom therapy relies on the ability of immunoglobulins to bind, extract, and neutralize toxins present in the body. It is most effective when produced from venom of locally endemic snakes and is used within appropriate time period, dosage and for appropriate indications, all of which play vital role in determining the outcomes of the therapy [17]. The most widely available ASV in Pakistan include, the Liquid ASV, locally produced by the National Institute of Health Islamabad and lyophilized/ liquid ASV, imported mostly from India [18]. The initial starting dose of $\mathrm{NIH}$ ASV against confirmed saw scale viper bite is four vials (40 mL) [18].

This study aims to determine the efficacy and safety of Antivenin manufactured by Anti-Snake Venom (ASV)/Anti-Rabies (ARV) Serology Laboratory, Health Department, Government of Sindh, in local patients of Sindh envenomed by Echis Carinatus Sochureki (Lundi).

\section{MATERIALS AND METHODS}

\section{Study Population}

This prospective observational study conducted on six patients referred to the emergency ward of Peoples University of Medical \& Health Sciences Hospital,

Table 1: Demographic Data of All Patients

\begin{tabular}{|c|c|c|c|c|}
\hline SN & Patients Code & Gender & Age (Years) & Weight (kg) \\
\hline \hline 1 & FAA & Male & 36 & 45 \\
\hline 2 & FAB & Male & 20 & 46 \\
\hline 3 & FAC & Male & 30 & 46 \\
\hline 4 & FAD & Female & 25 & 52 \\
\hline 5 & FAE & Male & 24 & 49 \\
\hline 6 & FAF & Male & 27.5 & 47.16667 \\
\hline
\end{tabular}

Table illustrates the demographic information of six (6) included patients with assigned codes, ages and weights.

Relevant Hospital Record regarding six included patients is available at project official website at http://asvsindh.gos.pk/human-clinical-trials-of-sindh-anti-snakevenom/ 
Nawabshah and District Headquarter Hospital Mithi, Sindh, Pakistan. Patients diagnosed as envenomed by Echis carinatus sochureki snake species. Out of six patients, five were males while one was a female, all belonged to local rural population. All patients aged 27.5 years \pm 2.06 years; weighing $47.16 \pm 1.03 \mathrm{~kg}$ (Table 1). All 6 Patients satisfied the inclusion criteria and were enrolled through a consecutive sampling technique. Inclusion criteria was very well documented in the study protocol and incorporated in the case report form. All patients were administered with $10 \mathrm{~mL}$ of ASV diluted in $100 \mathrm{~mL}$ NS.

\section{Materials}

Bivalent anti-venom was used to treat the patients envenomed by Echis carinatus sochureki snake species. It is a sterile preparation containing Gamma globulin antibodies with capability to neutralize the venom of Saw Scaled Viper and Russell's viper. These antibodies are obtained from the blood plasma of horses immunized with the venom of cited snakes, manufactured by ASVIARV Serology Laboratory, Health Department, Government of Sindh.

The chemicals used for ASV production were Caprylic Acid (Sigma), $\mathrm{NaOH}, \mathrm{NaCl}$, Citric Acid and Phenol (all Merck). Venom of Echis carinatus sochureki and Daboia russelli were obtained from the Toxinology Center, Institute of Biochemistry, Sindh University Jamshoro. The filtration assemblies were of Millipore (USA) and the micro and ultra-filters used were of Merck and Synder USA brand.

\section{Methods}

Patients referred to emergency wards of Peoples University of Medical \& Health Sciences Hospital, Nawabshah and District Headquarter Hospital Mithi, Sindh, Pakistan were enrolled in the study after confirmation of coagulopathy by 20 -minute WBCT. The patients were specifically asked and investigated for any spontaneous bleeding in past or for any disease likely to affect blood coagulation. All patients were administered with a standard ASV dose; investigations for Complete blood count and Urine detailed report were conducted before and after ASV dosing, Serum Creatinine levels were assessed as well. All patients were routinely monitored by clinical investigators assigned in the specific clinical facility for adverse events until the recovery from coagulopathy. As per hospital protocol for the management of snakebite, certain preceding medications were administered to patients. Details of demographic information (age, sex and weight), findings of physical examination including site of bite, local reaction at the bitten site and systemic features, and medical investigations records inclusive of complete blood count, urine detailed report and coagulation profile were noted. The coagulopathy was defined by the 20-minute WBCT which shows incoagulable blood twenty minutes after blood drawing [19]. Patients were routinely monitored for coagulopathy on 3, 6, 12 and 24 hours after ASV administration and subsequently discharged by attending physician (clinical investigator) after complete recovery from coagulopathy. Duration of exposure to study drug was the total time span starting from the administration of initial dose until the end of management dose. Evaluation of the safety of the product was routinely monitored by clinical investigators (specialists of the particular subjects working as faculty of Medical University acknowledge the internationally accepted protocols). They clinically observed the patients for any succeeding untoward effect every 3 hours after ASV dosing till the discharge of the patient as described in IRB approved study protocol.

The patients under trial were read upon with a statement approved by Institutional Review Board and Ethical Committee on Clinical Trials of Peoples University of Medical \& Health Sciences for Women Nawabshah to let the patient know the objectives and procedures of the clinical trial and its likely risks and benefits for the patient. An IRB approved draft consent farm was filled and got signed by the patient and accompanying relative before including the patient in the study.

\section{Registration}

Available on WHO International Clinical Trial Registry Platform, through Iranian Registry of Clinical Trials (IRCT) with main ID IRCT2014070218314N1

Search Portal http://apps.who.int/trialsearch/Trial2. aspx?TrialID=IRCT2014070218314N1

\section{Statistical Analysis}

Descriptive statistical technique was used. Software 'Solver' and Data-Analysis Tool-Pack of Microsoft Excel ${ }^{\circledR}$ were employed for all statistical work. Data is expressed as mean, median, and standard deviation.

\section{RESULTS}

All patients envenomed by Echis carinatus sochureki (Lundi) snake species and showed 
Table 2: Time of Complete Recovery of Coagulopathy after Initial Test ASV Dosing in Individual Patients

\begin{tabular}{|c|c|c|c|c|c|}
\hline SN & Subject Code & $\begin{array}{c}\text { Date of } \\
\text { administration }\end{array}$ & $\begin{array}{l}\text { Time for end of } \\
\text { management dose }\end{array}$ & $\begin{array}{c}\text { Time of Complete } \\
\text { recovery }\end{array}$ & $\begin{array}{l}\text { Time for Complete } \\
\text { recovery* (H:min) }\end{array}$ \\
\hline \multirow[t]{2}{*}{1} & FAA M.Dose** & $15 / 08 / 2015$ & 05:15:00 PM & \multirow[t]{2}{*}{ 10:26:00 PM } & \multirow[t]{2}{*}{$>24: 00$} \\
\hline & S. Dose $\mathrm{e}^{\star \star *}$ & $16 / 08 / 2015$ & 07:25:00 PM & & \\
\hline 2 & FAB & $16 / 08 / 2015$ & 01:50:00 PM & 10:28:00 PM & $08: 30$ \\
\hline 3 & FAC & $16 / 08 / 2015$ & 03:50:00 PM & 10:30:00 PM & $06: 40$ \\
\hline 4 & FAD & 09/09/2015 & 05:15:00 AM & 09:15:00 AM & 04:00 \\
\hline 5 & FAE & $11 / 09 / 2015$ & 05:00:00 PM & $12: 15: 00$ AM & $07: 15$ \\
\hline 6 & FAF & $17 / 09 / 2015$ & 05:30:00 PM & 08:30:00 PM & 03:00 \\
\hline & & & & Mean & 09:15 \\
\hline & & & & Median & 6.975 \\
\hline & & & & STDEV & 8.46 \\
\hline
\end{tabular}

Table illustrates the time of complete recovery of coagulopathy after receiving initial test ASV dose in individual patients.

*Time of complete recovery of coagulopathy after ASV dosing.

**Management Dose.

***Subsequent Dose.

coagulopathy on 20-minute WBCT. The investigational drug Bivalent Anti venom Immunoglobulin-NQ1 (IgG) was given as IV infusion under the supervision of attending physician (clinical investigator). $10 \mathrm{~mL}$ ASV diluted in $100 \mathrm{~mL}$ NS was administered to five of the patients while one was initially given $5 \mathrm{~mL}$ test dose and then $5 \mathrm{~mL}$ subsequent dose. Patients were routinely monitored for any adverse events and all were found to be recovered from coagulopathy uneventful. Treatment success was $100 \%$ as all study patients completely recovered from coagulopathy; mean recovery time was 9:15 $\pm 3: 25$ hours while time period required for individual patient's recovery is presented in Table 2. Mean duration of exposure was found to be $3: 22 \pm 0.22$ hours. No clinical or haematological adverse or seriously adverse effect was observed after Bivalent Anti venom Immunoglobulin-NQ1 (IgG) administration.

\section{DISCUSSION}

Snakebite injuries are not so uncommon public health emergencies especially encountered in rural and suburban areas of Asia [20-23].

Immunotherapy by Anti-venoms is the only effective approach in preventing death from snakebites; however, the type of anti-venom needed depends on the type of snake involved in the bite. Furthermore, anti-venoms frequently produce side effects [17]. In the absence of precise knowledge about the type of snake, anti-venom is given often based on the types known to be in the area [24]. In some parts of the world, getting the right type of anti-venom is difficult and expensive partly contributing to inefficiency of the administered antivenin.

The Bivalent Anti venom Immunoglobulin-NQ1 (IgG) manufactured by Anti Snake Venom (ASV)/ AntiRabies (ARV) Serology Laboratory, Health Department, Government of Sindh is produced from snake species present in the habitat of local region. The results show that the study drug Bivalent Anti venom Immunoglobulin-NQ1 (IgG) produced and complete its pharmacological effects within 6-12 hours. An infusion of $10 \mathrm{~mL}$ ASV diluted in $100 \mathrm{~mL}$ normal saline was administered. First patient FAA was treated by initial $5 \mathrm{~mL}$ ASV dose but according to response coagulopathy recovered after $10 \mathrm{~mL}$ ASV dosing for all patients. Our study demonstrates that only one vial (10 $\mathrm{mL}$ ) of Bivalent Anti venom Immunoglobulin-NQ1 (lgG) is effective in curing the envenoming by Echis Carinatus Sochureki as compared to $60-200 \mathrm{~mL}$ of other available anti snake venom preparation [25]. As Echis Carinatus Sochureki (saw scaled viper) specie produces haemolytic effects i.e. coagulopathy [26]. All patients had thrombocytopenia upon admission to the hospital; however, none of them presented with clinical signs of haemorrhagic because all reached within seven hours of snakebite. 20-minute WBCT test was carried out to confirm the diagnosis of snakebite coagulopathy as well as for assessing the recovery. All 6 Patients were routinely monitored on 3, 6, 12 and 24 hour of ASV dsoing for the recovery of coagulopathy through 20-minute WBCT test. They were also clinically monitored before and after ASV dosing by clinical investigators. Patients' recovery from coagulopathy 
after antivenin therapy was without any adverse events. No early or late adverse reaction to anti-venom therapy was observed.

This observational study investigated the effectiveness and adverse reactions to bivalent antivenom $\lg G$ in curing the snakebite victims of specie found in Sindh. As per results of this study (Phase II) safety and efficacy was developed for the Bivalent Anti venom Immunoglobulin-NQ1 (IgG) manufactured by Anti-Snake Venom(ASV)/Anti-Rabies (ARV) Serology Laboratory, Health Department, Government of Sindh . Further phase III comparative trials can be pursued against widely Used Polyvalent snake anti Venom; manufactured by National Institute of Health, Islamabad, Pakistan. Being an underdeveloped province of Pakistan, Sindh with highest number of snakebite cases suffers most from the shortage of Antivenom availability [27].

\section{POTENTIAL LIMITATIONS}

Small sample size: Apparently the sample size of snake bite patients studied looks very few to conclude any concrete result, but keeping in view the emergency situation of a patient in a life threatening condition, having anxiety about his / her survival it is very intricate for the clinician / clinical investigators to gain consent of the patient fulfilling all inclusion criteria. It is therefore the lowest acceptable number choosen, however the results acheived will further be accertained by similar trials in shape of comprative study and phase IV trials.

Open label nature: The study was conducted for assessing efficacy and safety of a newly produced ASV hence open label prefered as this has very little to no effect on the primary outcome of the study.

Lack of comparative arm: Due to special nature of trial the comparative arm kept limited to avoid any grevious effect to any patient under study. Moreover it was ethically impractical while managing a snakebite case to use any placebo to compare the effect of treatment.

Limited laboratory investigations. It could be supported with more confirmative laboratory investigations like asssessment of fibrinogen or other clotting factors but the part of clinical trial conducted at secondary care hospitals where majority of the snakebite cases are initially received but are having only basic laboratory facilities hence reliance kept on some basic blood tests including complete picture, platelet count, $\mathrm{Hb} \%$ and the 20 minute whole blood clotting time test which are workable in a developing country like Pakistan.

\section{ACKNOWLEDGEMENTS}

The ASVIARV Serology Laboratory is funded by the Provincial Health Department, Government of Sindh, Pakistan.

\section{AUTHOR CONTRIBUTIONS}

Naeem Quraishi Ph.D. is the chief investigator for the clinical trial, Tasneem Ahmad Ph.D. worked as associate investigator and quality assurer, Allah Bux Ghanghro Ph.D was responsible for bio-analytical procedures, Abdullah Arejo Ph.D. was responsible for production and testing on animals and Syed Muhammad Sharib Pharm.D. worked as research assistant during clinical trial.

\section{FINANCIAL / MATERIAL SUPPORT}

The study is funded by the Provincial Health Department, Government of Sindh, Pakistan through Research \& Development funds of the Establishment of ASVIARV Serology Laboratory Project.

\section{DISCLOSURE}

No obvious conflict of interest exists, as the project is not an income generation or business venture and is part of public sector health delivery system of the province. The mass production of the ASV under trial will be provided free of cost through government health care outlets.

\section{REFERENCES}

[1] Chippaux J-P. Estimating the global burden of snakebite can help to improve management. PLoS Med 2008; 5(11): e221. https://doi.org/10.1371/journal.pmed.0050221

[2] Kasturiratne A, Wickremasinghe AR, de Silva N, et al. The global burden of snakebite: a literature analysis and modelling based on regional estimates of envenoming and deaths. PLoS Med 2008; 5(11): e218. https://doi.org/10.1371/journal.pmed.0050218

[3] Auerbach PS, Norris RL. Disorders caused by reptile bites and marine animal exposures. Harrisons Principles of Internal Medicine 2005; 16(2): 2593.

[4] Suchithra N, Pappachan JM, Sujathan P. Snakebite envenoming in Kerala, South India: clinical profile and factors involved in adverse outcomes. Emergency Medicine Journal 2008; 25(4): 200-204.

https://doi.org/10.1136/emj.2007.051136

[5] Hansdak SG, Lallar KS, Pokharel P, Shyangwa P, Karki P, Koirala S. A clinico-epidemiological study of snake bite in Nepal. Tropical Doctor 1998; 28(4): 223-226.

https://doi.org/10.1177/004947559802800412 
[6] Chandio AM, Sandelo P, Rahu AA, Ahmed ST, Dahri AH, Bhatti R. Snake bite: Treatment seeking behavior among Sindh rural population. JAMC 2000; 12(3).

[7] Agarwal R, Aggarwal AN, Gupta D, Behera D, Jindal SK. Low dose of snake antivenom is as effective as high dose in patients with severe neurotoxic snake envenoming. Emergency Medicine Journal 2005; 22(6): 397-399. https://doi.org/10.1136/emj.2004.020727

[8] Ilyas M. Community meden, $4^{\text {th }}$ ed. 1997.

[9] http://asvsindh.gos.pk/project-digest/provincial-situation/

[10] Warrell DA. Clinical toxicology of Snake bites in Asia. In: White MA, editor. Handbook of clinical toxicology of animal venoms and poisons. CRC Press 1995; pp. 493-588.

[11] Warrell DA. Snake venoms in science and clinical medicine. 1. Russell's viper: biology, venom and treatment of bites. Trans R Soc Trop Med Hyg 1989; 83: 732-740. https://doi.org/10.1016/0035-9203(89)90311-8

[12] Ansari AK, Sheikh SA. Management of Vipride Snake Bite. Pak Armed Forces Med J 2000; 50(1): 26-28

[13] Warrell DA. Poisoning by bites of the saw-scaled or carpet viper [Echis carinatus] in Nigeria. QJM 1977; 46(1): 33-62

[14] Sitwat HA, et al. Study of snake bite cases at Liaquat university hospital Hyderabad/Jamshoro. J Ayub Med Coll Abbottabad 2008; 20(3): 125-127.

[15] Hunter GW, Strickland GT, Eds. Hunter's tropical medicine and emerging infectious diseases. WB Saunders company, 2000; Vol. 857.

[16] Gutiérrez JM, Lomonte B, Leon G, Rucavado A, Chaves F, Angulo $\mathrm{Y}$. Trends in snakebite envenomation therapy: scientific, technological and public health considerations. Current Pharmaceutical Design 2007; 13(28): 2935-2950. https://doi.org/10.2174/138161207782023784

[17] Warrell DA. The clinical management of snake bites in the Southeast Asian region. Southeast Asian J Trop Med Public Health 1999; 30(Suppl 1): 1-67.

[18] Quraishi NA, Qureshi HI, Simpson ID. A contextual approach to managing snake bite in Pakistan: snake bite treatment with particular reference to neurotoxieity and the ideal hospital snake bite kit. JPMA. The Journal of the Pakistan Medical Association 2008; 58(6): 325-331.

[19] Ahmed SM, Ahmed M, Nadeem A, Mahajan J, Choudhary A, Pal J. Emergency treatment of a snake bite: Pearls from literature. Journal of Emergencies, Trauma and Shock 2008; 1(2): 97.

https://doi.org/10.4103/0974-2700.43190

[20] Viravan C, Looareesuwan S, Kosakarn W, et al. A national hospital-based survey of snakes responsible for bites in Thailand. Transactions of the Royal Society of Tropical Medicine and Hygiene 1992; 86(1): 100-106. https://doi.org/10.1016/0035-9203(92)90463-M

[21] Pandey DP. Epidemiology of snakebites based on field survey in Chitwan and Nawalparasi districts, Nepal. Journal of Medical Toxicology 2007; 3(4): 164-168. https://doi.org/10.1007/BF03160933

[22] Hutton RA, Looareesuwan S, Ho M, Silamut K, et al. "Arboreal green pit vipers [genus Trimeresurus] of SouthEast Asia: bites by T. albolabris and T. macrops in Thailand and a review of the literature. Transactions of the Royal Society of Tropical Medicine and Hygiene 1990; 84(6): 866874.

\section{https://doi.org/10.1016/0035-9203(90)90111-Q}

[23] Bhardwaj A, Sokhey J. Snake bites in the hills of north India. National Medical Journal of India 1998; 11(6).

[24] Animal bites Fact sheet $N^{\circ} 373$. World Health Organization.

[25] Zafar J, Aziz S, Hamid B, Qayyum A, Alam MT, Qazi RA. Snake bite: Experience at Pakistan Institute of Medical Sciences. Journal- Pakistan Medical Association 1998; 48: 308-310.

[26] Lakhotia M, Pahadiya HR, Singh J, Gandhi R, Bhansali S. Lakhotia, Manoj, et al. Adrenal hematoma and right hemothorax after echis carinatus bite: An unusual manifestation. Toxicology International 2014; 21(3): 325. https://doi.org/10.4103/0971-6580.155388

[27] http://asvsindh.gos.pk/project-digest/the-regional-situation

Received on 03-03-2017

DOI: https://doi.org/10.6000/1927-5951.2017.07.03.5

(c) 2017 Quraishi et al.; Licensee Lifescience Global.

This is an open access article licensed under the terms of the Creative Commons Attribution Non-Commercial License (http://creativecommons.org/licenses/by-nc/3.0/) which permits unrestricted, non-commercial use, distribution and reproduction in any medium, provided the work is properly cited. 